Doug Geisler, Eva K. Grebel, and Dante Minniti, eds.

\title{
The Globular Cluster System of NGC 5128
}

\author{
Marina Rejkuba \\ ESO, Karl-Schwarzschild-Strasse 2, Garching bei München, Germany \\ and P. Universidad Católica de Chile, Santiago, Chile
}

\begin{abstract}
On the basis of high resolution ground-based images, taken with VLT and FORS1, 71 bona fide globular clusters were discovered in the halo of NGC 5128. Only 5 of these clusters were previously known. In a UV color-magnitude diagram for the complete sample of 71 clusters and a $(\mathrm{U}-\mathrm{V})_{0}$ vs. $\left(\mathrm{V}-\mathrm{K}_{s}\right)_{0}$ color-color diagram for a subsample of 23 clusters, the objects span a similar magnitude and color range as the globular clusters in the MW and M31. The GCLFs spanning $-10.1<M_{V}<-4.9$ and $-9.3<M_{U}<-3.3$ have been constructed. These are the deepest GCLFs of in a $\mathrm{gE}$ made so far. The Kolmogorov-Smirnov statistics show that GCLF of the giant elliptical NGC 5128 is consistent with the ones of spiral galaxies like the MW and M31.
\end{abstract}

\section{Introduction}

The globular cluster luminosity function (GCLF) of a galaxy is the relative number of its globular clusters per luminosity (or magnitude) interval. It is usually unimodal and nearly symmetric with a peak at a characteristic (turnover) magnitude. Empirically it was found that turn-overs for galaxies at the same distance (e.g. in the same galaxy cluster) have internal dispersion of < 0.1, which makes GCLF a good standard candle (e.g. Harris 1991). The full calibration of the GCLF, however, is incomplete. The essential problem is that the galaxies at large distances which are the most attractive targets for the GCLF method are giant ellipticals in which globular clusters are found in largest numbers, whilst the primary calibration of the standard candle $M_{V}^{0}$ rests on the MW and M31, which are spiral galaxies.

\section{Globular clusters in NGC 5128}

I used VLT Antu (UT1) telescope with FORS1 to obtain high resolution images of two fields in the halo of the nearest $(\mathrm{d} \sim 3.6 \mathrm{Mpc})$ giant elliptical galaxy NGC 5128. The Field 1 is centered on the prominent diffuse north-eastern shell, approximately $14 \mathrm{kpc}$ away from the center of the galaxy and Field 2 is in the halo $9 \mathrm{kpc}$ away from the center. In addition, $\mathrm{K}_{s}$-band image of Field 2 was obtained with SOFI at NTT at La Silla Observatory.

Images were processed within IRAF environment. After the subtraction of the overscan and bias and the division by the flat-field image, the photometry of all the sources was performed with DAOPHOT package in IRAF. 

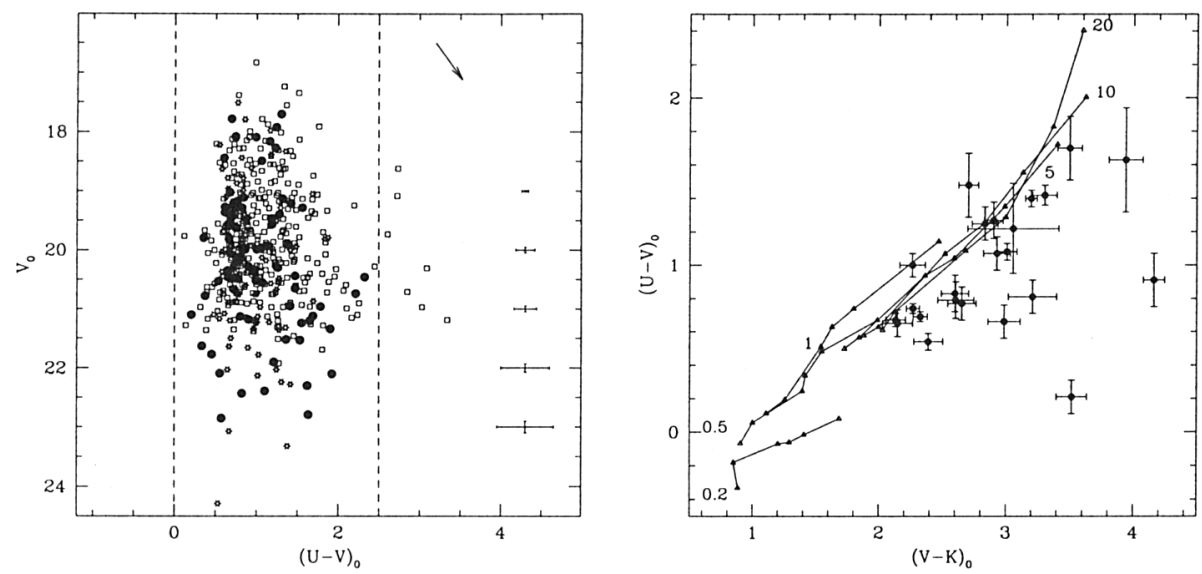

Figure 1. Left: The comparison of the UV CMDs of NGC 5128 globular clusters (full dots) with the ones of the Milky Way (stars) and M31 (squares). Right: Color-color diagram for globular clusters in NGC 5128. The lines are the isochrones of SSP models (Kurth et al. 2000) for $\mathrm{Z}=0.0001,0.0004,0.008,0.004,0.02 \mathrm{ad} 0.05$. The number next to each isochrone indicates the age in Gyr.

NGC 5128 is close enough so that its globular clusters can be distinguished from stars on the basis of their slightly non-stellar appearance even from the ground with high resolution imaging in excellent seeing conditions. Because of their slightly larger FWHM and non-stellar PSF, globular clusters were easily detected on residual images created by subtracting the objects fitted with a stellar PSF. Globular cluster residuals were oversubtracted in the center and undersubtracted in the wings.

In order to be classified as a bona fide globular cluster, the object had to (i) show non-stellar PSF that leaves ring-like residuals on subtracted images; (ii) have a FWHM larger than the stellar FWHM and ellipticity $<0.4$; and (iii) have $0<(U-V)_{0}<2.5$. The selection of objects with the ellipticity measured on the V-band images smaller than 0.4 excludes galaxies from the sample. The color range in the last criterion corresponds to the observed range of the $(\mathrm{U}-\mathrm{V})_{0}$ colors for the MW and M31 globular clusters.

The comparison of the color-magnitude diagrams (CMD) of NGC 5128 globular clusters with the ones in the Milky Way and M31 is given in the left panel of Fig. 1. In this comparison the assumed distance modulus of for NGC 5128 is 27.8 (Soria et al. 1996) and for M31 24.47. The mean reddening towards NGC 5128 is $E(B-V)=0.1$. In the right panel the $(U-V)_{0}$ vs. $(V-K)_{0}$ color-color diagram is compared to the simple stellar population models from Kurth et al. (2000). Unfortunately the models do not allow to disentangle ages in the range of 5-20 Gyr. However, it is comforting to see that almost all the objects are concentrated around the isochrones in excess of $1 \mathrm{Gyr}$. This is another evidence that they are indeed globular clusters. 

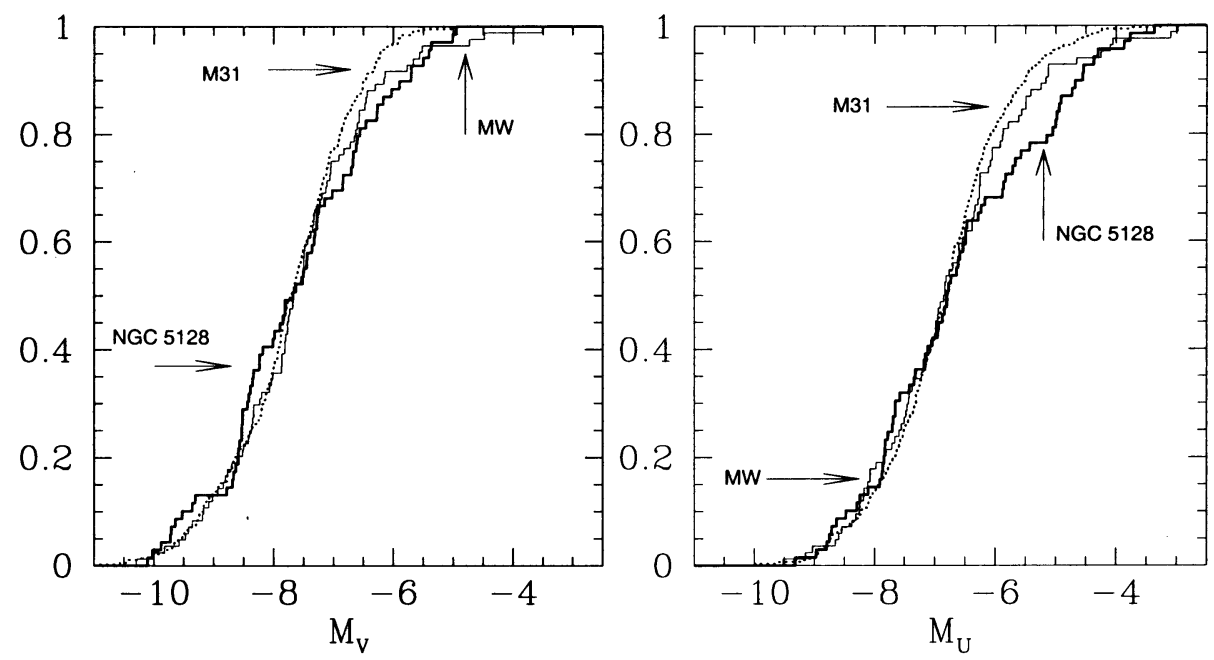

Figure 2. The cumulative distribution function for NGC 5128 (thick line) is constructed from the V-band GCLF (left panel) and U-band GCLF (right panel) and compared with the ones of the MW (thin line) and M31 (dotted line).

\section{The Universal GCLF}

The GCLFs spanning $-10.1<M_{V}<-4.9$ and $-9.3<M_{U}<-3.3$ have been constructed. These are the deepest GCLFs of an elliptical galaxy made so far.

The actual shape of the luminosity function depends on the size of the bins and on the bin-centers. Statistically it is possible to compare them independently from the binning with the Kolmogorov-Smirnov test. The Kolmogorov-Smirnov statistics shows that the difference between the GCLFs of NGC 5128 and MW is not larger than the difference between the GCLFs of M31 and MW (Fig. 2). Similarity of the GCLFs of an elliptical with respect to the spiral galaxy had never been tested at the faint end before.

\section{References}

Harris, W.E. 1991, ARA\&A 29, 543

Kurth, O.M., Fritze-v. Alvensleben, U. \& Fricke, K.J. 1999, A\&AS, 138, 19

Soria, R., Mould, J.R., Watson, A.M., et al. 1996, ApJ, 465, 79 